\title{
Specific Anthocyanin Contents of Whole Blue Maize Second-Generation Snacks: An Evaluation Using Response Surface Methodology and Lime Cooking Extrusion
}

\author{
Anayansi Escalante-Aburto, ${ }^{1,2,3}$ Néstor Ponce-García, ${ }^{1,4}$ Benjamín Ramírez-Wong,' \\ Patricia Isabel Torres-Chávez, ${ }^{1}$ Juan de Dios Figueroa-Cárdenas, ${ }^{2}$ \\ and Roberto Gutiérrez-Dorado ${ }^{5}$ \\ ${ }^{1}$ DIPA, Universidad de Sonora, Boulevard Luis Encinas y Rosales $s / n, 83000$ Hermosillo, SON, Mexico \\ ${ }^{2}$ CINVESTAV-Unidad Querétaro, Libramiento Norponiente No. 2000, Fraccionamiento Real de Juriquilla, \\ 76230 Querétaro, QRO, Mexico \\ ${ }^{3}$ Universidad de Monterrey, Avenida Ignacio Morones Prieto No. 4500 Poniente, 66238 San Pedro Garza García, NL, Mexico \\ ${ }^{4}$ UAEMex, Campus Universitario "El Cerrillo", El Cerrillo Piedras Blancas s/n, 50200 Toluca, MEX, Mexico \\ ${ }^{5}$ Programa Regional del Noroeste para el Doctorado en Biotecnología, Universidad Autónoma de Sinaloa, \\ Avenida de Las Américas y Boulevard Universitarios s/n, 80010 Culiacán, SIN, Mexico \\ Correspondence should be addressed to Benjamín Ramírez-Wong; bramirez@guaymas.uson.mx
}

Received 24 September 2015; Accepted 3 January 2016

Academic Editor: Patricia Valentao

Copyright (C) 2016 Anayansi Escalante-Aburto et al. This is an open access article distributed under the Creative Commons Attribution License, which permits unrestricted use, distribution, and reproduction in any medium, provided the original work is properly cited.

\begin{abstract}
Lime cooking extrusion (LCE) is a widely applied technology for producing second-generation snacks, as an alternative to traditional nixtamalization (TN). Pigmented maize has been used to produce snacks with similar organoleptic characteristics to TN products and to obtain a product with additional functional benefits due to the anthocyanic compounds contained in those grains. However, during the process, anthocyanins are degraded, and several chemical modifications occur. Response surface methodology is applied to evaluate extrusion factors and their effects on the response variables of extrudates. The aim of this study was to evaluate the changes in specific anthocyanins after extrusion in second-generation blue maize snacks. Three anthocyanins were identified and quantified by HPLC-UV-DAD: cyanidin 3-glucoside and pelargonidin 3-glucoside, which have been previously reported in blue maize and its products, and cyanidin 3,5-diglucoside. Higher retention values were found in the extrudates making LCE a viable option for producing second-generation blue maize snacks.
\end{abstract}

\section{Introduction}

Anthocyanins represent the major group of water-soluble pigments in the plant kingdom and are widely distributed in food crops (e.g., vegetables, roots, tubers, and cereals). Several investigations have reported not only nontoxic and nonmutagenic effects but also therapeutic and antioxidant activities of anthocyanic compounds. The aglycones (basic forms) of anthocyanins are termed anthocyanidins. These structures are based on the flavylium ion or 2-phenyl benzopyrylium, which presents hydroxyl and methoxyl groups in different positions [1]. Anthocyanidins are not accumulated as such but rather in a glycosylated form, that is, linked to sugars, in which case they are known as anthocyanins. The sugars attached to anthocyanidins provide high solubility and stability. Generally, the sugar molecule is linked to the phenolic acid at the 3 position, although it can be linked at the 5 and 7 positions as well [2]. Cyanidin 3-glucoside has been reported as the most abundant anthocyanin contained in pigmented maize (approximate from $48 \%$ up to $87 \%$ ), but pelargonidin 3-glucoside, peonidin 3-glucoside, and malvidin 3-glucoside can also be present [3]. The pigmented maize cultivated in 
TABLE 1: Experimental design of LCE showing different combinations of FM, T, and LC and experimental values of anthocyanin contents of the extrudates.

\begin{tabular}{|c|c|c|c|c|c|c|}
\hline \multirow{4}{*}{$\mathrm{Tx}^{1}$} & FM & \multirow{3}{*}{$\begin{array}{c}\mathrm{T} \\
{ }^{\circ} \mathrm{C} \\
\text { Process factors }\end{array}$} & \multirow{3}{*}{$\begin{array}{c}\mathrm{LC} \\
\%\end{array}$} & \multirow{2}{*}{\multicolumn{2}{|c|}{$\begin{array}{r}\mathrm{C} 3 \mathrm{G} \\
\mathrm{mg} \cdot \mathrm{kg}^{-1}\end{array}$}} & \multirow[t]{3}{*}{ Pel3G } \\
\hline & $\%$ & & & & & \\
\hline & & & & \multicolumn{2}{|c|}{ Response variables } & \\
\hline & $X_{1}$ & $X_{2}$ & $X_{3}$ & $Y_{1}$ & $Y_{2}$ & $Y_{3}$ \\
\hline 1 & $19(0)$ & $130(0)$ & $0.13(0)$ & 16.98 & 28.10 & 1.63 \\
\hline 2 & $15(-1.682)$ & $130(0)$ & $0.13(0)$ & 16.47 & 27.22 & 1.71 \\
\hline 3 & $19(0)$ & $130(0)$ & $0.13(0)$ & 17.09 & 28.17 & 1.72 \\
\hline 4 & $19(0)$ & $130(0)$ & $0.13(0)$ & 17.06 & 27.61 & 1.66 \\
\hline 5 & $21.38(1)$ & $141.89(1)$ & $0.2(1)$ & 16.97 & 28.07 & 1.73 \\
\hline 6 & $19(0)$ & $130(0)$ & $0.13(0)$ & 17.03 & 28.21 & 1.76 \\
\hline 7 & $19(0)$ & $130(0)$ & $0.25(1.682)$ & 17.29 & 28.36 & 1.75 \\
\hline 8 & $21.38(1)$ & $118.11(-1)$ & $0.05(-1)$ & 16.89 & 27.41 & 1.59 \\
\hline 9 & $19(0)$ & $130(0)$ & $0(-1.682)$ & 16.75 & 27.77 & 1.78 \\
\hline 10 & $21.38(1)$ & $141.89(1)$ & $0.05(-1)$ & 16.92 & 27.96 & 1.72 \\
\hline 11 & $16.62(-1)$ & $141.89(1)$ & $0.05(-1)$ & 16.58 & 27.26 & 1.70 \\
\hline 12 & $16.62(-1)$ & $118.11(-1)$ & $0.2(1)$ & 16.48 & 26.67 & 1.64 \\
\hline 13 & $23(1.682)$ & $130(0)$ & $0.13(0)$ & 16.84 & 27.64 & 1.53 \\
\hline 14 & $21.38(1)$ & $118.11(-1)$ & $0.2(1)$ & 16.89 & 27.48 & 1.69 \\
\hline 15 & $19(0)$ & $110(-1.682)$ & $0.13(0)$ & 16.69 & 27.18 & 1.61 \\
\hline 16 & $16.62(-1)$ & $141.89(1)$ & $0.2(1)$ & 16.40 & 26.96 & 1.70 \\
\hline 17 & $19(0)$ & $150(1.682)$ & $0.13(0)$ & 16.65 & 27.23 & 1.67 \\
\hline 18 & $16.62(-1)$ & $118.11(-1)$ & $0.05(-1)$ & 16.59 & 26.97 & 1.64 \\
\hline 19 & $19(0)$ & $130(0)$ & $0.13(0)$ & 16.70 & 27.26 & 1.53 \\
\hline 20 & $19(0)$ & $130(0)$ & $0.13(0)$ & 16.69 & 27.26 & 1.47 \\
\hline
\end{tabular}

${ }^{1}$ Numbers in parentheses are the coded levels. Tx: treatment; FM: feed moisture; T: temperature; LC: lime concentration; C3,5-diG: cyanidin 3,5-diglucoside; C3G: cyanidin 3-glucoside; Pel3G: pelargonidin 3-glucoside.

American and Asiatic countries contains high amounts of these compounds; however, during processing, anthocyanins degrade due to high $\mathrm{pH}$, temperature, and light exposure [4].

Extrusion is a technology widely used to obtain different maize products, such as expanded snacks. Lime cooking extrusion (LCE) is considered a faster and effective alternative method to traditional nixtamalization (TN) processing, the latter based on the addition of $\mathrm{Ca}(\mathrm{OH})_{2}$ to maize and water at $100^{\circ} \mathrm{C}$ to form a solution that is cooked for $20-40 \mathrm{~min}$ and steeped for at least $16 \mathrm{~h}$ [5]. LCE processing involves thermal treatment, low moisture, and high-pressure conditions, among other factors (incorporation of additives and conditioning of flours). Many studies [6, 7] have been conducted on pigmented maize using LCE to retain more anthocyanins, limiting the losses due to processing. However, none of these studies have analyzed the profile of anthocyanins as being affected by multiple process factors using response surface methodology (RSM), a statistical method used to investigate the effects of factors in complex processes, and examined how these factors influence the different anthocyanins contained in the extrudates. The aim of this study was to evaluate the changes in specific anthocyanin contents that occurred in extruded products elaborated with LCE, applying RSM.

\section{Materials and Methods}

2.1. Raw Material. Blue maize was obtained from a local market in Toluca, Mexico. The grains were cleaned and stored at $5^{\circ} \mathrm{C}$ in black polyethylene bags until use. Commercial lime (Calhidra de Sonora, SA de CV, Hermosillo, SON, Mexico) and distilled water were used.

\subsection{Methods}

2.2.1. Lime Cooking Extrusion (LCE). The process was performed according to the method described by EscalanteAburto et al. [4]. Ten kilograms of blue maize was passed through a blade mill (Pulvex SA de CV, Model 200, serial 1030401, Mexico, DF) with a $0.8 \mathrm{~mm}$ mesh. Twelve hours before the extrusion experiment, the ground maize was divided into 20 batches of $300 \mathrm{~g}$, and commercial lime and distilled water were added to each batch according to the experimental design shown in Table 1.

To obtain the extrudates, the experiment was performed in a single screw extruder (Brabender Instruments, Model E19/25 D, OHG Duisburg, Germany). The temperatures in the first, second, and third zones were set at 60, 80, and $110^{\circ} \mathrm{C}$, respectively, and the fourth zone varied from $110^{\circ} \mathrm{C}$ to $150^{\circ} \mathrm{C}$ (Table 1). The screw had a diameter of $19 \mathrm{~mm}$, and the nominal compression ratio was $3: 1$. The other extrusion conditions were a screw speed of $120 \mathrm{rpm}$, feed hopper of $50 \mathrm{rpm}$, and die opening diameter of $4 \mathrm{~mm}$. The extrudates were cooled at room temperature and stored in sealed bags until the chemical analyses were performed. 


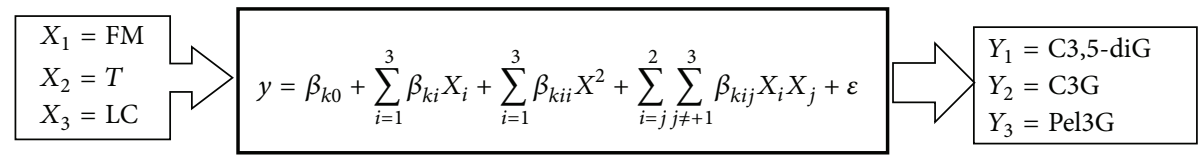

FIGURE 1: Empirical black box modeling of the process variables and the response variables.

2.2.2. Anthocyanin Profile by HPLC Analysis. The extraction of anthocyanins was based on the methodology of AbdelAal and Hucl [8]. Extrudate samples were ground (Moulinex, model 980-18, France) and passed through a number 60 sieve. Two $\mathrm{mg}$ of sample was weighed into a $50 \mathrm{~mL}$ centrifuge tube (tube 1) with $20 \mathrm{~mL}$ of acidified ethanol (ethanol $+\mathrm{HCl}$ $1 \mathrm{~N}, 85: 15 \mathrm{v} / \mathrm{v}$ ) and agitated (Wrist Action Shaker, model 75, Burrell Corp., EUA) for $1 \mathrm{~h}$. Then, the solution was centrifuged (Thermo Scientific, model Heraeus Biofuge Primo R., Germany) at $27,200 \times \mathrm{g}$ for $15 \mathrm{~min}$, and the supernatant was recovered in a $50 \mathrm{~mL}$ tube. Twenty $\mathrm{mL}$ of acidified ethanol was added to the residue contained in tube 1 , and the above agitation and centrifugation steps were repeated. The supernatants from both steps were combined, giving an approximate volume of $40 \mathrm{~mL}$, and then concentrated to a final volume of $10 \mathrm{~mL}$ in a nitrogen evaporator $(\mathrm{N}$ EVAP $^{\text {mox }} 112$, Organomation Associates, Inc., EUA) at $40^{\circ} \mathrm{C}$. The extracts were passed through a membrane of $0.45 \mu \mathrm{m}$ (Merck, Millipore, Darmstadt, Germany) and stored at $2^{\circ} \mathrm{C}$ in amber vials until analysis. Three extracts from each treatment were obtained.

An HPLC instrument with UV-vis and a DAD detector (Agilent Technologies, model 1260 Infinity, Germany) was used. The separation was performed in a reverse-phase C18 column (Waters, model Spherisorb ODS-2, $5 \mu \mathrm{m}, 250$ $\times 46 \mathrm{~mm}$, Ireland). The mobile phases were A, methanol, and $\mathrm{B}$, formic acid (5\%). The gradient used was as follows: 0-30 min 25\% A, $75 \%$ B; $31-60$ min $40 \%$ A, $60 \%$ B; flow rate, $1.5 \mathrm{~mL} / \mathrm{min}$; column temperature, $35^{\circ} \mathrm{C}$; detection wavelength, $535 \mathrm{~nm}$; injection volume, $20 \mu \mathrm{L}$. The analyses were performed in triplicate.

Standards of five specific anthocyanins, cyanidin 3,5diglucoside (cyanidin chloride), cyanidin 3-glucoside (kuromanin chloride), cyanidin 3-galactoside (idaein chloride), pelargonidin 3-glucoside (callistephin chloride), and malvidin 3-glucoside (oenin chloride), were prepared, and calibration curves were obtained for concentrations from 0.025 to $1.0 \mathrm{mg} / \mathrm{mL}$.

2.3. Experimental Design and Statistical Analysis. A central composite experimental design of three factors and five levels was chosen (Table 1). The independent variables were feed moisture, FM $\left(X_{1}, 15 \%-23 \%\right)$; lime concentration, LC $\left(X_{2}\right.$, $0 \%-0.25 \%)$; and fourth-zone extruder temperature, $T\left(X_{3}\right.$, $110-150^{\circ} \mathrm{C}$ ), coded with levels of $-1.682,-1,0,+1$, and +1.682 . The response variables were cyanidin 3,5-diglucoside (C3,5$\left.\mathrm{diG}, \mathrm{mg} \cdot \mathrm{kg}^{-1}\right)$, cyanidin 3-glucoside $\left(\mathrm{C} 3 \mathrm{G}, \mathrm{mg} \cdot \mathrm{kg}^{-1}\right)$, and pelargonidin 3-glucoside (Pel3G, $\mathrm{mg} \cdot \mathrm{kg}^{-1}$ ). The empirical model representing the interaction between the independent and response variables is presented in Figure 1.
This mathematical expression is used to model the response variables $\left(Y_{1}-Y_{3}\right)$, where the $k$ value changes from 1 to $4 ; \beta k_{0}$ represents a constant, $\beta k_{i}$ the linear coefficient, $\beta k_{i i}$ the quadratic coefficient, $\beta k_{j i}$ the interaction effect of the response variables, and $\varepsilon$ the experimental error. The response variable data were recorded, and an analysis of variance (ANOVA) was performed with a confidence level of 95\%. Moreover, a backward regression analysis was applied, and nonsignificant factors $(P>0.1)$ were eliminated from the second-order polynomial equation. Then, a new equation was recalculated to achieve the final predictive model for each response variable. RSM was used [9] and contour plots for each determination were obtained using Design Expert Software V.7.0.0.

\section{Results and Discussion}

HPLC analyses demonstrated that of the five anthocyanins analyzed only three were present in raw blue maize and its extrudates. Cyanidin 3,5-diglucoside, cyanidin 3-glucoside, and pelargonidin 3-glucoside were identified. C3,5-diG was the first anthocyanin eluted. The glucose molecules affected the polarity, degree of glycosylation, and nature of the sugar moiety, and thus the elution order was galactoside $>$ glucoside $>$ arabinoside [10]. Moreover, the presence of multiple glycosidic substituents affected the retention characteristics, provoking a faster elution of the compounds, as in the case of C3,5-diG.

3.1. Effects of FM, T, and LC on C3,5-Diglucoside Concentration. The coefficients of the second-order equations, ANOVA, and determination coefficients of the effects of FM, $T$, and LC on the C3,5-diG are shown in Table 2.

The results demonstrated that FM in its linear and quadratic $(\mathrm{FM})^{2}$ terms exerts highly significant $(P<0.0024)$ and significant $(P<0.0200)$ effects, respectively, on the C3,5diG concentration. In addition, temperature in its quadratic term $(T)^{2}$ presented a very significant effect $(P<0.092)$ on the anthocyanin. The prediction model equation was as follows:

$$
\mathrm{C} 3,5-\mathrm{diG}=-3.92+0.81(\mathrm{HA})-0.01(\mathrm{HA})^{2}-0.0007(T)^{2} .
$$

Table 1 displays the average values of the concentration of C3,5-diG, which varied from $16.40 \mathrm{mg} \cdot \mathrm{kg}^{-1}$ to $17.29 \mathrm{mg} \cdot \mathrm{kg}^{-1}$. C3,5-diG was the second most abundant compound after C3G. The latter situation could occur because diglycosylated anthocyanins have higher stability than monoglycosylated ones, which diminishes the susceptibility to degradation by temperature and UV radiation [11]. Moreover, when the 
TABLE 2: Coefficients of second-order equations (prediction models) and analysis of variance, showing the relationship between process factors and response variables in the extrudates.

\begin{tabular}{cccc}
\hline Coefficient & C3G & C3,5-diG & Pel3G \\
\hline $\begin{array}{c}\text { Intercept } \\
\beta\end{array}$ & 27.78 & 16.93 & 1.63 \\
Linear & & & \\
$\beta_{1}$ & $0.28^{*}$ & $0.16^{* * *}$ & -0.018 n.s. \\
$\beta_{2}$ & 0.13 n.s. & -0.003 n.s. & 0.029 n.s. \\
$\beta_{3}$ & 0.042 n.s. & 0.50 n.s. & -0.003 n.s. \\
Quadratic & & & \\
$\beta_{11}$ & -0.16 n.s. & $-0.11^{* *}$ & -0.002 n.s. \\
$\beta_{22}$ & $-0.24^{*}$ & $-0.10^{*}$ & -0.002 n.s. \\
$\beta_{33}$ & 0.061 n.s. & 0.020 n.s. & $0.049^{*}$ \\
Interactions & & & \\
$\beta_{12}$ & 0.070 n.s. & 0.026 n.s. & -0.005 n.s. \\
$\beta_{13}$ & 0.098 n.s. & 0.042 n.s. & 0.013 n.s. \\
$\beta_{23}$ & 0.006 n.s. & -0.001 n.s. & -0.009 n.s. \\
\hline
\end{tabular}

C3G, cyanidin 3-glucoside; C3,5-diG, cyanidin 3,5-diglucoside; Pel3G, pelargonidin 3-glucoside; $\beta_{1}$, feed moisture; $\beta_{2}$, final extruder temperature; $\beta_{3}$, lime concentration; n.s., not significant $(P>0.1) ;{ }^{*}$ significant $(P<0.1)$; ${ }^{* *}$ very significant $(P<0.05) ;{ }^{* * *}$ highly significant $(P<0.01)$.

retention of $\mathrm{C} 3,5-\mathrm{diG}$ in the extrudates was evaluated in comparison to the values obtained in raw blue maize, it was found that between 91.2 and $96.2 \%$ of the anthocyanin was retained in the products. The stability of $\mathrm{C} 3,5-\mathrm{diG}$ to the processing conditions applied in this investigation was demonstrated. Our results differ from the findings of Žilić et al. [12], as the blue maize used in this study contained 10 times more C3,5-diG (17.98 $\left.\mathrm{mg} \cdot \mathrm{kg}^{-1}\right)$ than the concentrations reported by those authors, which were 0.08 and $1.65 \mathrm{mg} \cdot \mathrm{kg}^{-1}$ for multicolored and light blue maize kernels, respectively. According to Méndez et al. [5], the genotype, genetic antecedents, physical properties, and relation of pericarp and endosperm (structures that concentrate the phenolic compounds) affected the anthocyanin content of the maize grains.

Figure 2(a) shows the effects of FM and $T$ on the C3,5diG concentration of extrudates. It was observed that in a range of $21-23 \% \mathrm{FM}$ and at $\mathrm{T}$ of 120 and $130^{\circ} \mathrm{C}$ the anthocyanin reached its maximum concentration. When the $\mathrm{HA} *$ LC interaction was analyzed (Figure 2(b)), a directly proportional effect of FM and $\mathrm{C} 3,5-\mathrm{diG}$ was observed: as FM increases, the C3,5-diG content of the extrudates also increases, independently of LC.

Salinas Moreno et al. [13] reported the presence of C3G, Pel3G, peonidin 3-glucoside, three acylated anthocyanins, cyanidin-3-(6" malonylglucoside), cyanidin3-( $3^{\prime \prime}, 6^{\prime \prime}$ dimalonylglucoside), and four unidentified anthocyanins. This investigation demonstrated that C3,5diglucoside was present in blue maize and its extrudates.

Figure 2(c) showed the effect of the $T *$ LC interaction on C3,5-diG concentration. Only $T$ significantly affected this response variable, and the maximum average values of $\mathrm{C} 3,5-$ diG were found at $130^{\circ} \mathrm{C}$.
3.2. Effects of FM, T, and LC on the Cyanidin 3-Glucoside (C3G) Concentration. The coefficients of the second-order equations and ANOVA of the effect of FM, T, and LC are shown in Table 2. The FM presented a very significant effect in the linear and quadratic terms, while $T$ in the quadratic term $(T)^{2}$ showed a very significant effect $(P<0.0195)$ on the C3G values. The prediction model equation was as follows:

$$
\mathrm{C} 3 \mathrm{G}=-16.56+1.25(\mathrm{FM})-0.029(\mathrm{FM})^{2}-0.001(T)^{2} .
$$

The average values of $\mathrm{C} 3 \mathrm{G}$ were in the range of 26.67 to $28.36 \mathrm{mg} \cdot \mathrm{kg}^{-1}$ (Table 1). There were slight losses of these anthocyanins, as the extrudates retained between 77.1 and $82 \%$ of C3G compared with raw blue maize $\left(34.60 \mathrm{mg} \cdot \mathrm{kg}^{-1}\right)$. Figure 3(a) shows the effects of FM and $T$ on the $\mathrm{C} 3 \mathrm{G}$ content. Increased $\mathrm{FM}$ and $\mathrm{T}$ of $130^{\circ} \mathrm{C}$ resulted in the highest concentrations of these compounds. The interaction $\mathrm{FM} * T$ did not show a significant effect; nevertheless, there were significant effects only of the individual terms, meaning that the results could be related to the findings reported by Yue and $\mathrm{Xu}$ [14]. These authors concluded that although anthocyanins degraded at temperatures higher than $100^{\circ} \mathrm{C}$, thermal stability increased in compounds with glucose or galactose substituents. Moreover, there was a significant production of anthocyanidins such as delphinin and malvidin (present in pigmented maize) when their corresponding anthocyanins were submitted to heating at $100^{\circ} \mathrm{C}$ for 30 minutes in conditions of very low moisture.

Figure 3(b) shows the effects of FM and LC. A significant effect of FM can be observed; as these process factors increased, the concentration of C3G grew higher. It has been reported that $\mathrm{LC}$ and $\mathrm{pH}$ affect the anthocyanin concentration. In an alkaline medium, anthocyanin compounds diminish in content or are completely destroyed. However, there were no significant effects of LC. Figure 3(c) shows the effects of temperature and LC. Between $130^{\circ} \mathrm{C}$ and $140^{\circ} \mathrm{C}$, the $\mathrm{C} 3 \mathrm{G}$ content in the extrudates was higher, independently of LC. Sánchez-Madrigal et al. [15] evaluated the effect of LC addition on the concentration of $\mathrm{C} 3 \mathrm{G}$ extracted from blue maize nixtamalized flours obtained by extrusion. Those authors concluded that the concentration of C3G increases without significant effects from the calcium source added.

3.3. Effects of FM, T, and LC on Pelargonidin 3-Glucoside (Pel3G) Concentration. The coefficients of the second-order equations, ANOVA, and determination coefficients of the effects of FM, T, and LC on Pel3G concentration in the extrudates are shown in Table 2. The results demonstrated that LC in its quadratic term (LC) ${ }^{2}$ presented a significant effect $(P<0.0273)$ on Pel3G concentration. The prediction model equation was as follows:

$$
\text { Pel3G }=1.76-2.15(\mathrm{LC})+8.79(\mathrm{LC})^{2} .
$$

Table 1 displays the average values of Pel3G in the extrudates, which are in a range of $1.47-1.78 \mathrm{mg} \cdot \mathrm{kg}^{-1}$. Low amounts of these compounds were lost in the extrudates compared with raw blue maize $\left(2.29 \mathrm{mg} \cdot \mathrm{kg}^{-1}\right)$, as between 64.1 and $77.7 \%$ of Pel3G was retained in the products. The Pel3G 


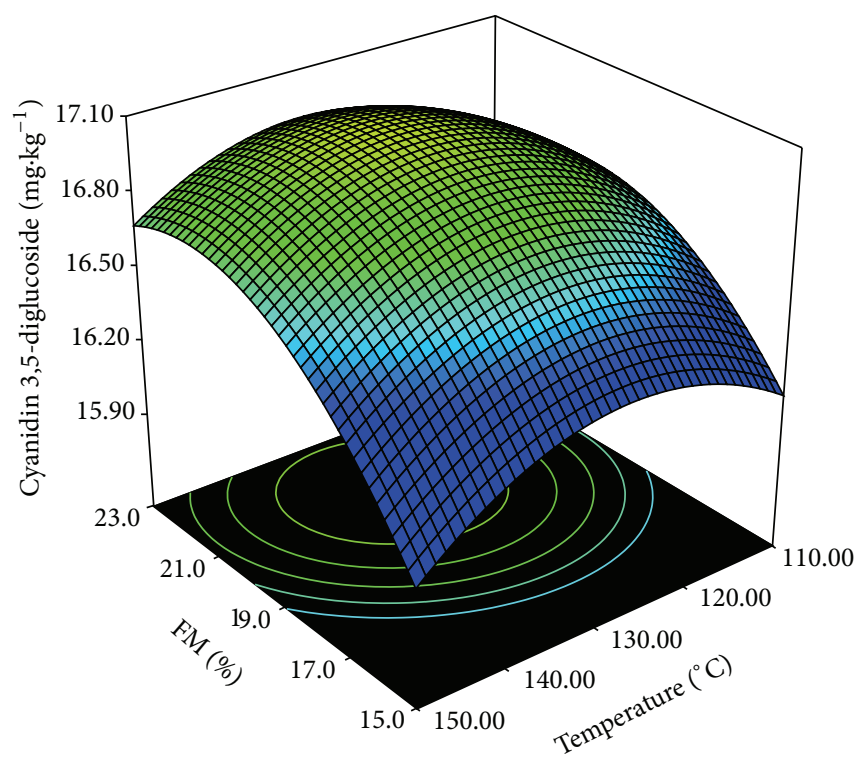

(a)

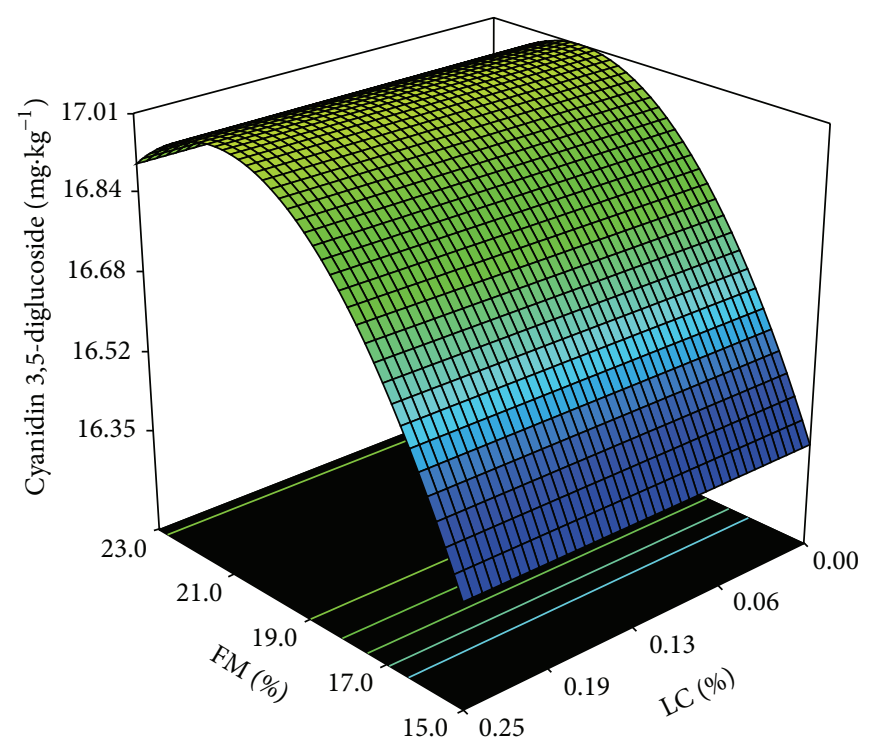

(b)

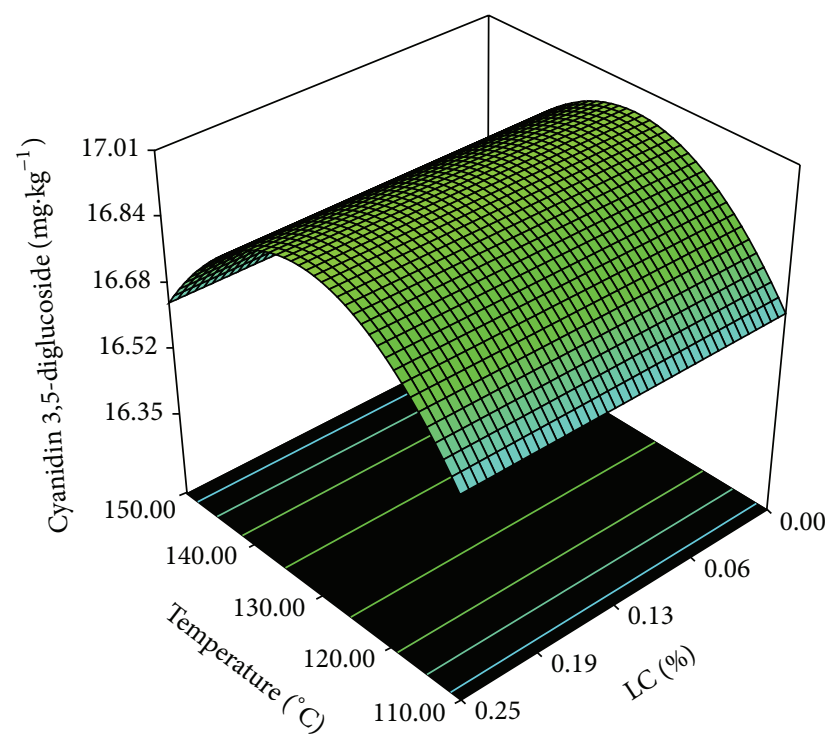

(c)

FIGURE 2: Effects of the processing factors (a) FM *T, (b) FM $* \mathrm{LC}$, and (c) $T *$ LC on C3,5-diG content in extruded blue maize snacks.

concentration in the extrudates was higher than that reported by Sánchez-Madrigal et al. [15] in blue maize nixtamalized flours obtained by extrusion processing. This result corroborates the different chemical transformations of anthocyanic compounds during processing, in addition to the differences in the anthocyanin profile for each maize variety, despite the same grain coloration. Even when Pel3G was present at minimum concentrations, it had a high stable chemical structure.

Garzón and Wrolstad [16] reported that Pel3G exerted a very significant effect on the degradation velocity of anthocyanin contained in certain food products with these compounds added, due to the higher stability to thermal processing.

Figure 4(a) shows the effect of the FM $* T$ interaction on Pel3G concentration in the extrudates. Maximum concentrations of these compounds were found at $130^{\circ} \mathrm{C}$ and low levels of FM (14\%), which agreed with the work by Garzón and Wrolstad [16] with respect to anthocyanin stability at higher processing temperatures.

When the effect of the FM $*$ LC interaction was analyzed (Figure 4(b)), it was observed that, at minimum and maximum LC ( $0-0.25 \%)$, Pel3G reached its highest concentration independently of FM. Figure 4(c) shows the effect of the $T *$ 


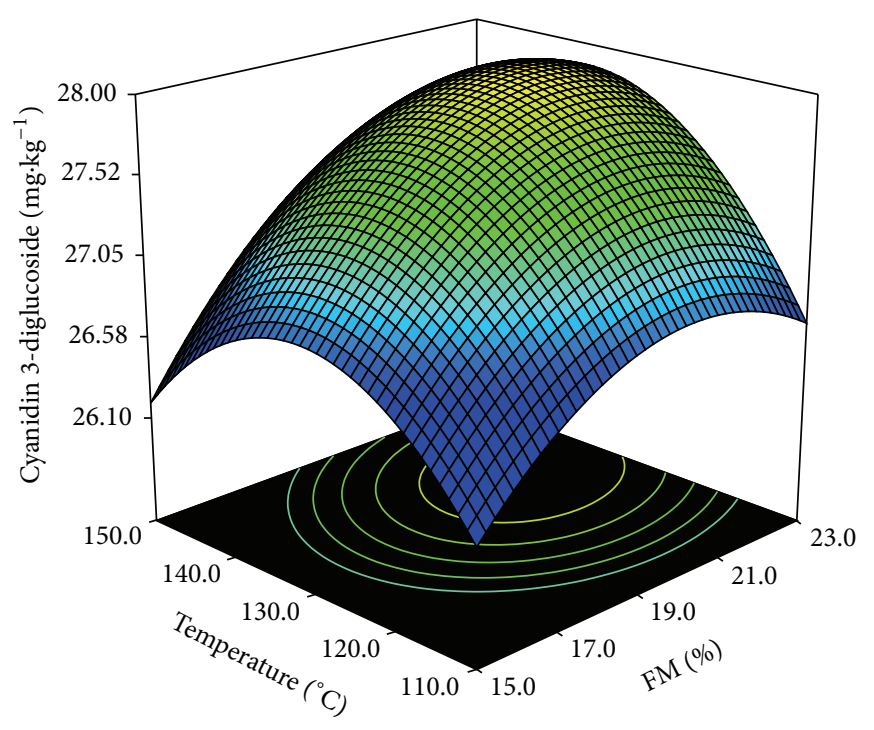

(a)

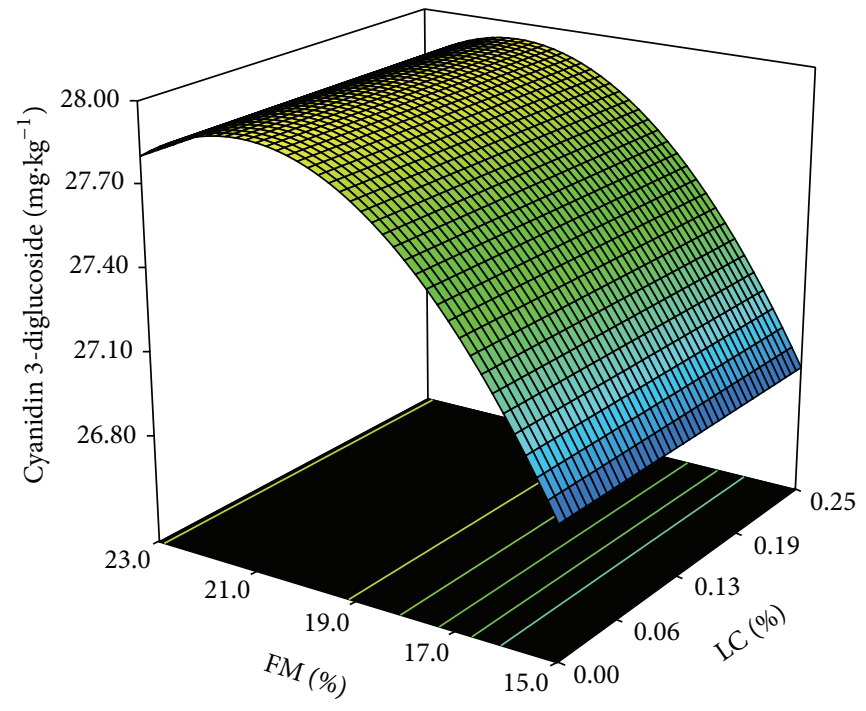

(b)

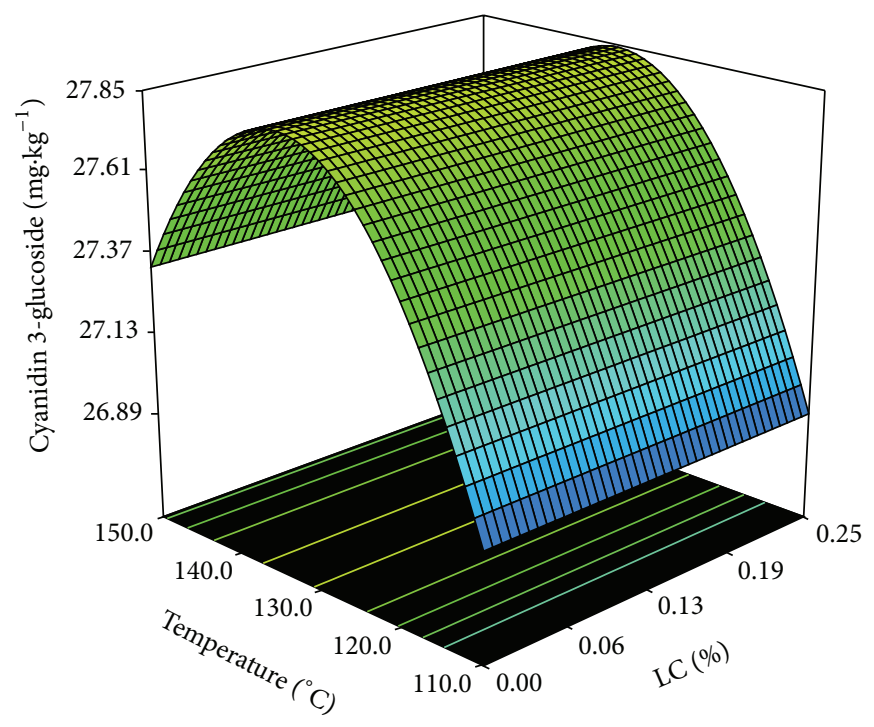

(c)

FigURE 3: Effects of the processing factors (a) $\mathrm{FM} * T$, (b) $\mathrm{FM} * \mathrm{LC}$, and (c) $T *$ LC on C3G content in extruded blue maize snacks.

LC interaction, where Pel3G presented higher degradation at LC $0.05-0.16 \%$ and $T 110-120^{\circ} \mathrm{C}$.

Although the interaction of FM and $T$ did not show significant effects and only the individual effects were significant, our results could be related with the findings reported by Yue and $\mathrm{Xu}$ [14]. They found that even when anthocyanins were degraded at temperatures higher than $100^{\circ} \mathrm{C}$, the thermal stability of these compounds with glucose or galactose substituents was better. Moreover, higher production of anthocyanidins such as cyanidin, delphinin, and malvidin (present in pigmented maize) was observed when their anthocyanins were subjected to temperatures higher than $100^{\circ} \mathrm{C}$ and very low moisture conditions.
In general, the results indicated higher retention percentages due to the short time of residence of raw material, implying minimum conditions of thermal treatment and hence higher retention of pigments. Anthocyanins showed certain sensitivity to the individual and combined effects of processing, such as temperature, pressure, treatment time, and the mechanisms and kinetics of degradation [17]. As we can see in the models, Pel3G was the only anthocyanin that showed a significant effect of LC, which was correlated with the $\mathrm{pH}$. Andrés-Bello et al. [18] reported that, with increasing $\mathrm{pH}$, anthocyanins become paler in color; however, if the food matrix contains substances capable of acting as copigments, the color may be retained and stabilized to a certain extent. 


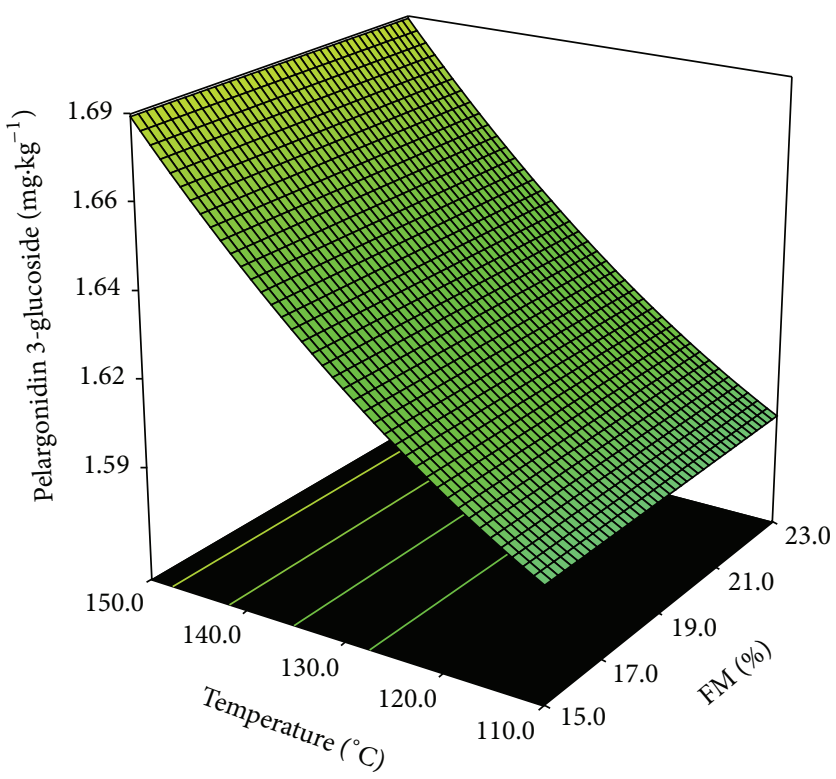

(a)

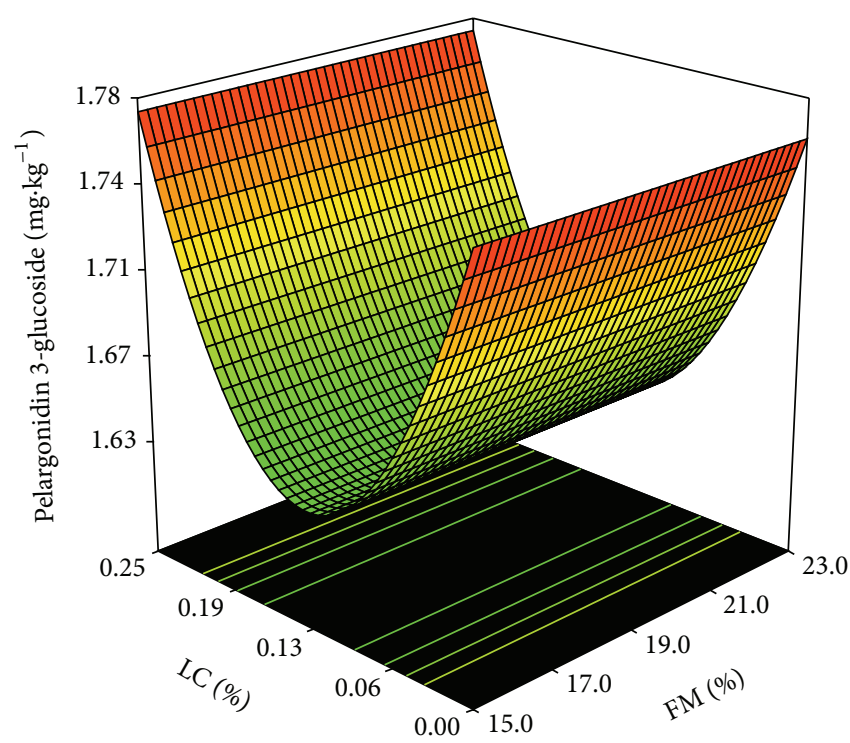

(b)

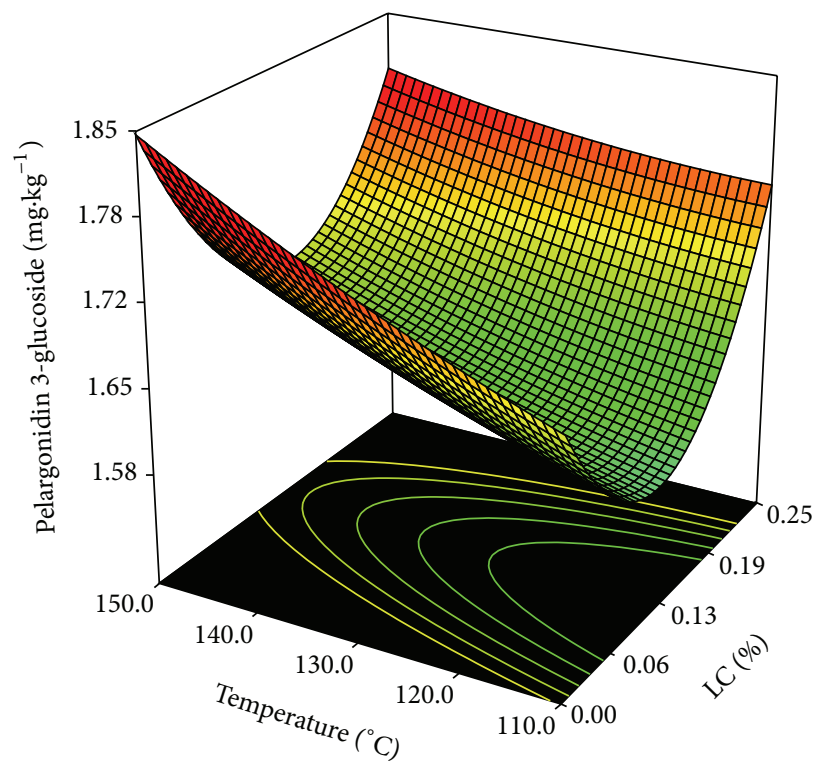

(c)

Figure 4: Effects of the processing factors (a) FM $* T$, (b) FM $*$ LC, and (c) $T *$ LC on Pel3G content in extruded blue maize snacks.

When anthocyanins are processed in an alkaline medium ( $\mathrm{pH} 7)$, the pericarp is not completely hydrolyzed by calcium hydroxide (lime), and the release of ferulic acid is lower. It has been reported that ferulic acid can act as a copigment of anthocyanins, increasing their retention during processing.

\section{Conclusions}

Higher retention percentages of anthocyanins were observed in the extruded snacks, even when an alkaline medium was induced with the lime cooking extrusion and the materials were exposed to high temperatures. Feed moisture, temperature, and their interactions showed significant effects on the anthocyanin content. Cyanidin 3-glucoside was the most abundant anthocyanin in the extruded snacks at the highest values at feed moisture contents (21-23\%) and temperatures on the fourth zone of the extruder $\left(130-140^{\circ} \mathrm{C}\right)$, with no significant effects of LC. Cyanidin 3,5-diglucoside due to its chemical composition showed higher stability and had the highest values at feed moisture contents of $21-23 \%$ and temperature of $130^{\circ} \mathrm{C}$, regardless of the LC. On the other hand, pelargonidin 3-glucoside was significantly affected by $\mathrm{LC}$, showing the highest values at temperature of $150^{\circ} \mathrm{C}$, and 
lime concentration range of $0-0.25 \%$, regardless of the feed moisture content. These results indicated that it is possible to produce snacks with beneficial or nutraceutical compounds using the lime cooking extrusion process.

\section{Conflict of Interests}

The authors declare that there is no conflict of interests regarding the publication of this paper.

\section{Acknowledgment}

Escalante-Aburto thanks CONACYT for the postdoctoral scholarship provided.

\section{References}

[1] M. Horbowicz, R. Kosson, A. Grzesiuk, and H. Dębski, "Anthocyanins of fruits and vegetables, their occurrence, analysis and role in human nutrition," Vegetable Crops Research Bulletin, vol. 68, no. 1, pp. 5-22, 2008.

[2] M. Y. Salinas, R. J. Soria, and T. E. Espinosa, Aprovechamiento y Distribución de Maíz Azul en el Estado de México, INIFAP, 1st edition, 2010.

[3] A. Castañeda-Ovando, M. D. L. Pacheco-Hernández, M. E. Páez-Hernández, J. A. Rodríguez, and C. A. Galán-Vidal, "Chemical studies of anthocyanins: a review," Food Chemistry, vol. 113, no. 4, pp. 859-871, 2009.

[4] A. Escalante-Aburto, P. I. Torres-Chávez, B. Ramírez-Wong, and N. Ponce-García, "Pigmented maizes: anthocyanin profile and content," in Handbook of Anthocyanins. Food Sources, Chemical Applications and Health Benefits, Nova Science Publishers, New York, NY, USA, 1st edition, 2015.

[5] L. I. R. Méndez, J. D. D. F. Cárdenas, M. R. Gómez, and L. L. M. Lagunas, "Nutraceutical properties of flour and tortillas made with an ecological nixtamalization process," Journal of Food Science, vol. 78, no. 10, pp. C1529-C1534, 2013.

[6] J. Aguayo-Rojas, S. Mora-Rochín, E. O. Cuevas-Rodríguez et al., "Phytochemicals and antioxidant capacity of tortillas obtained after lime-cooking extrusion process of whole pigmented mexican maize," Plant Foods for Human Nutrition, vol. 67, no. 2, pp. 178-185, 2012.

[7] A. Escalante-Aburto, B. Ramírez-Wong, P. I. Torres-Chávez et al., "Obtaining ready-to-eat blue corn expanded snacks with anthocyanins using an extrusion process and response surface methodology," Molecules, vol. 19, no. 12, pp. 21066-21084, 2014.

[8] E.-S. M. Abdel-Aal and P. Hucl, "A rapid method for quantifying total anthocyanins in blue aleurone and purple pericarp wheats," Cereal Chemistry, vol. 76, no. 3, pp. 350-354, 1999.

[9] R. H. Myers, D. C. Montgomery, and C. M. Anderson-Cook, "The analysis of second-order response surfaces," in Response Surface Methodology. Process and Product Optimization Using Designed Experiments, John Wiley \& Sons, Hoboken, NJ, USA, 3rd edition, 2009.

[10] V. Hong and R. E. Wrolstad, "Use of HPLC separation/photodiode array detection for characterization of anthocyanins," Journal of Agricultural and Food Chemistry, vol. 38, no. 3, pp. 708-715, 1990.

[11] M. T. Escribano-Bailón, C. Santos-Buelga, and J. C. RivasGonzalo, "Anthocyanins in cereals," Journal of Chromatography A, vol. 1054, no. 1-2, pp. 129-141, 2004.
[12] S. Žilić, A. Serpen, G. Akillioğlu, V. Gökmen, and J. Vančetović, "Phenolic compounds, carotenoids, anthocyanins, and antioxidant capacity of colored maize (Zea mays L.) kernels," Journal of Agricultural and Food Chemistry, vol. 60, no. 5, pp. 1224-1231, 2012.

[13] Y. Salinas Moreno, G. Salas Sánchez, D. Rubio Hernández, and N. Ramos Lobato, "Characterization of anthocyanin extracts from maize kernels," Journal of Chromatographic Science, vol. 43, no. 9, pp. 483-487, 2005.

[14] X. Yue and Z. Xu, "Changes of anthocyanins, anthocyanidins, and antioxidant activity in bilberry extract during dry heating," Journal of Food Science, vol. 73, no. 6, pp. C494-C499, 2008.

[15] M. Á. Sánchez-Madrigal, A. Quintero-Ramos, F. MartínezBustos et al., "Effect of different calcium sources on the bioactive compounds stability of extruded and nixtamalized blue maize flours," Journal of Food Science and Technology, vol. 52, no. 5, pp. 2701-2710, 2015.

[16] G. A. Garzón and R. E. Wrolstad, "Comparison of the stability of pelargonidin-based anthocyanins in strawberry juice and concentrate," Journal of Food Science, vol. 67, no. 4, pp. 1288-1299, 2002.

[17] A. V. Durge, S. Sarkar, and R. S. Singhal, "Stability of anthocyanins as pre-extrusion colouring of rice extrudates," Food Research International, vol. 50, no. 2, pp. 641-646, 2013.

[18] A. Andrés-Bello, V. Barreto-Palacios, P. García-Segovia, J. Mir$\mathrm{Bel}$, and J. Martínez-Monzó, "Effect of $\mathrm{pH}$ on color and texture of food products," Food Engineering Reviews, vol. 5, no. 3, pp. 158-170, 2013. 

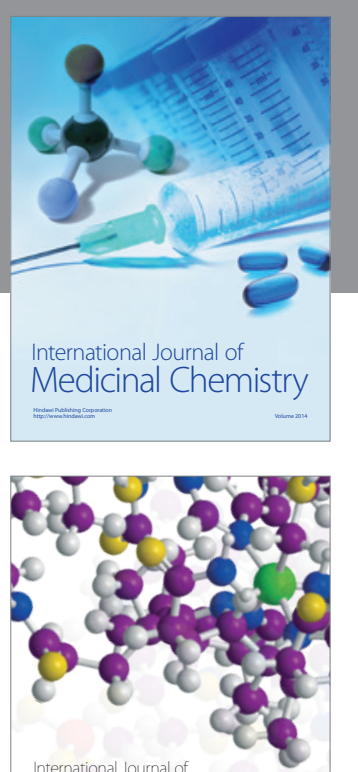

Carbohydrate Chemistry

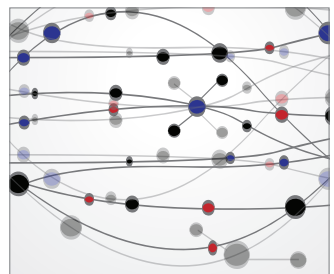

The Scientific World Journal
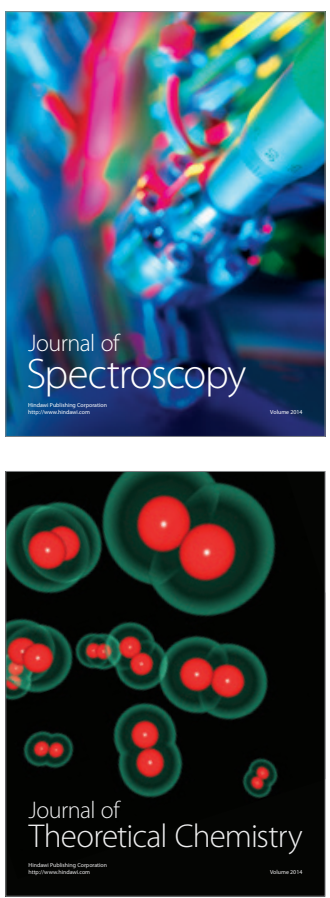
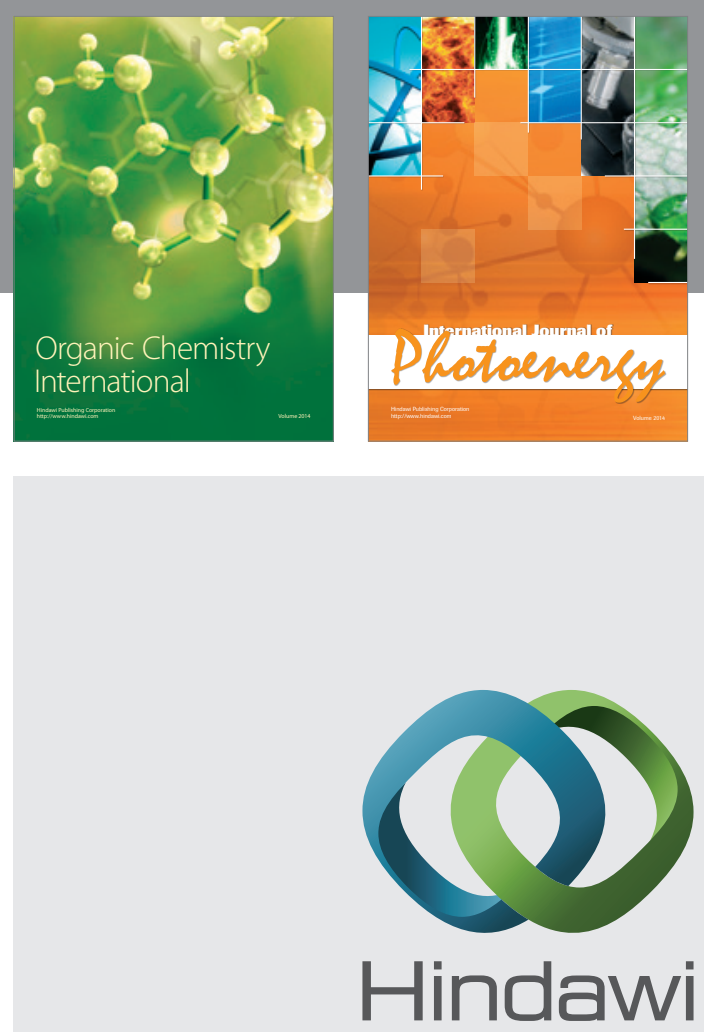

Submit your manuscripts at

http://www.hindawi.com

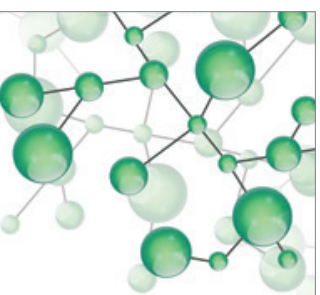

International Journal of

Inorganic Chemistry

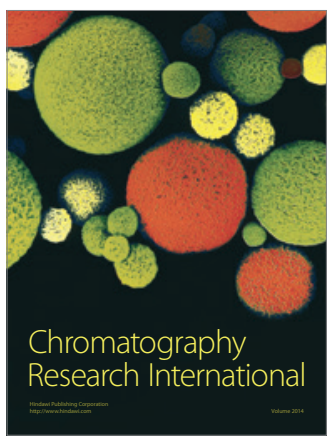

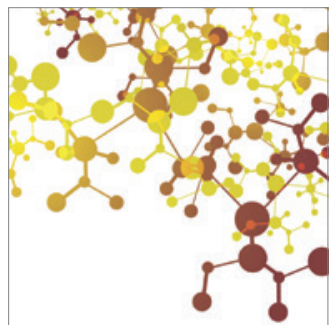

Applied Chemistry
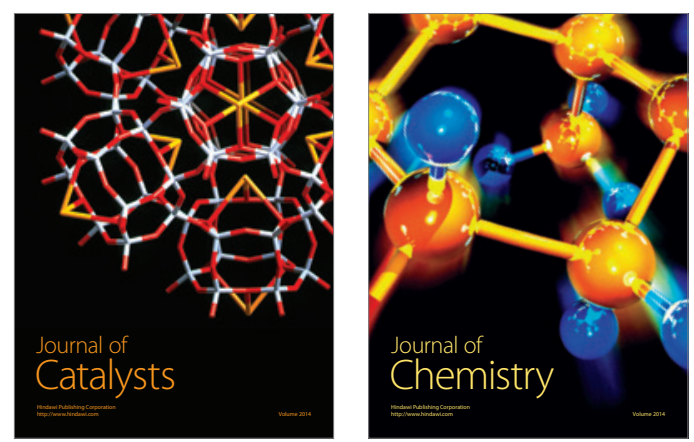
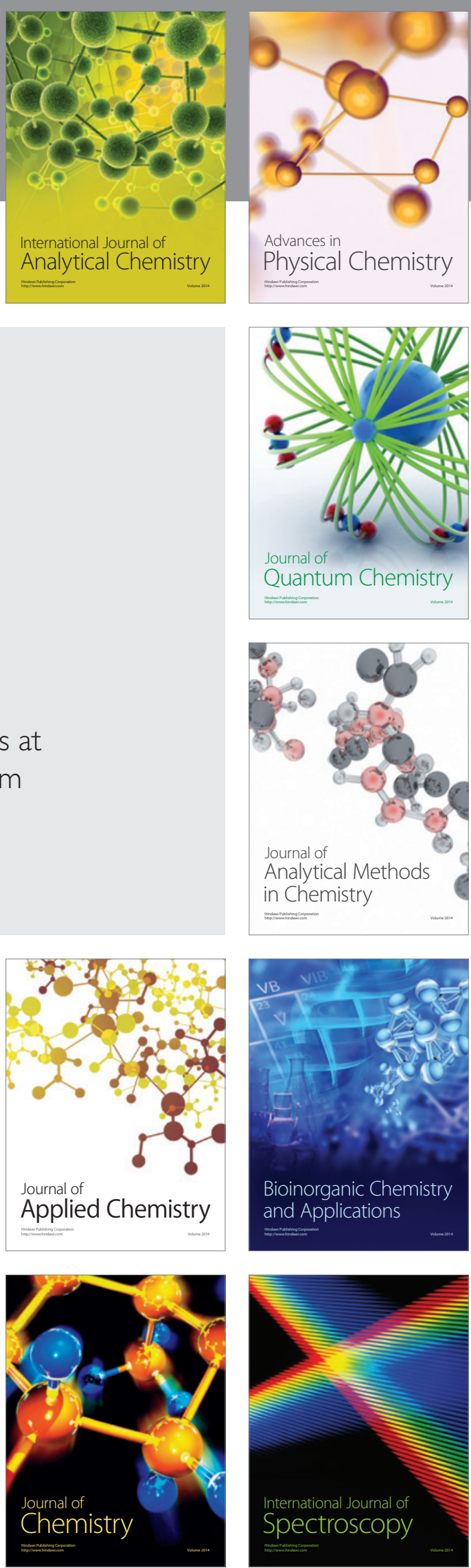